\title{
Extra-nasal methicillin-resistant Staphylococcus aureus colonization and maintenance hemodialysis patients
}

\author{
Helmut Schiffl ${ }^{*}$ \\ Department of Internal Medicine IV, University Hospital Munich. LMU-University, Munich, Germany
}

\section{A R T I C L E I N F O}

Article Type:

Epidemiology and prevention

\section{Article History:}

Received: 14 February 2019

Accepted: 23 April 2019

Published online: 31 May 2019

\section{Keywords:}

Staphylococcus aureus, Carriage, Hemodialysis, End-stage renal disease

\section{Implication for health policy/practice/research/medical education:}

Methicillin resistant Staphylococcus aureus (MRSA) infections of endogenous origin are important causes of morbidity and mortality in immunocompromised hemodialysis patients. MRSA colonization at extra-renal sites is increasingly recognized in patient subpopulations at risk. However, the prevalence of extrarenal MRSA colonization in dialysis patients is largely unknown. Of clinical importance, extra-nasal MRSA colonization predisposes to blood stream infections in hemodialysis patients with non-cuffed central vein catheter. Routine extra-nasal testing of hemodialysis patients should be recommended for successful decolonization and reduction of life-threatening infections.

Please cite this paper as: Schiffl H. Extra-nasal methicillin-resistant Staphylococcus aureus colonization and maintenance hemodialysis patients. J Nephropharmacol. 2019;8(2):e28. DOI: $10.15171 / \mathrm{npj} .2019 .28$
S taphylococcus aureus infections remain common in maintenance haemodialysis (HD) patients. The consequences of these infections, particularly blood stream infections with methicillin-resistant $S$. aureus (MRSA) are potentially severe and entail costly therapy. Patients with end-stage renal disease are exceedingly vulnerable to $S$. aureus infections for many reasons, including a) the immunosuppressed state of uremia and the high burden of comorbid diseases; b) the exposure to other patients or health care workers in the HD facility three times per week; c) frequent hospitalisations and antibiotic regimens; d) the invasive nature of the HD procedure (central venous catheter, arterio-venous fistula) and the high prevalence rates for S. aureus/MRSA colonization. Efforts specifically directed at the reduction of MRSA infection rates have focused on transmission dynamics and have included screening for nasal colonization, decolonization and barrier strategies (1). Numerous studies from around the world have demonstrated a high prevalence of nasal S. aureus/MRSA colonization in ESRD patients undergoing haemodialysis treatment. Nasal MRSA colonization of patients on extracorporeal maintenance $\mathrm{HD}$ has been associated with higher subsequent risk of infection/bacteraemia and repeated hospital admissions. MRSA nasal colonization may also be a marker for a more general vulnerability of individual HD patients and for an increased risk for allcause mortality (2).
A meta-analysis including 38 relevant studies (5596 dialysis patients) showed that the pooled prevalence of nasal MRSA colonization was about $7 \%$ of patients receiving regular haemodialysis therapy (3). However, nasal colonization varies with geographic region, patient characteristics, sampling methods (collection system, number of swabs) and detection techniques (direct plating or PCR).

False negative culture results may arise from sampling errors or insensitive collection methods. Moreover, longitudinal investigations utilizing two or more nasal swabs revealed three patterns of $S$. aureus or MRSA nasal carriage. They could be defined both in patients maintained on haemodialysis as well as for healthy adults as (1) persistent carriers, (2) intermittent carriers and (3) persistent non-carriers (1). True negative cultures can be found in non-carriers, but also in patients with intermittent MRSA carriage. Real time PCR is the only current method with a sensitivity approaching $100 \%$ (1). Extra-nasal S. aureus colonization (oropharynx, inguinal region, axilla, vascular access or other sites) is more common as currently assumed with a prevalence of approximately one third of all dialysis patients (4). In certain body sites it may be even more common than nasal colonization. Extra-nasal $S$. aureus carriage is as significant as nasal carriage for $\mathrm{S}$. aureus blood stream infections in patients on HD (5). There are only few data describing extra-nasal colonization with MRSA among 
maintenance haemodialysis patients. We found that $32 \%$ of patients with MRSA carriage were colonized in more than one body site $(2,6)$. Zahed et al reported a low extranasal MRSA colonization rate of $1.7 \%$. Extra-nasal MRSA screening in HD patients will increase MRSA detection by more than $30 \%$ compared with nares screening alone. Extra-nasal testing of HD patients may be a valuable strategy for outbreak control in a setting of persistent MRSA infection.

Eradication of MRSA carriage by decolonization and enhanced infection prevention protocols is a crucial clinical challenge, as it may reduce the risk of lifethreatening infection of colonized haemodialysis patients and prevent MRSA transmission to other patients who were not colonized. However, the success rate of the decolonization procedure may be high in patients with only nasal MRSA carriage, but significantly lower in patients with additional extra-nasal (wounds, throat) MRSA colonization (7). Nevertheless, to achieve MRSA decolonization we need to consider not just nasal decolonization but also decolonization of the skin and oropharynx.

\section{Author's contribution}

HS wrote the manuscript alone.

\section{Ethical considerations}

Ethical issues (including plagiarism, data fabrication, double publication) have been completely observed by the author.

\section{Conflicts of interest}

The author declares no conflict of interest.

\section{References}

1. Lederer SR, Riedelsdorf G, Schiffl H. Nasal carriage of meticillin resistant Staphylococcus aureus: the prevalence, patients at risk and the effect of elimination on outcomes among outclinic haemodialysis patients. Eur J Med Res. 2007;12:284-8.

2. Schmid H, Romanos A, Schiffl H, Lederer SR. Persistent nasal methicillin-resistant staphylococcus aureus carriage in hemodialysis outpatients: a predictor of worse outcome. BMC Nephrol. 2013;14:93. doi: 10.1186/1471-2369-14-93.

3. Zacharioudakis IM, Zervou FN, Ziakas PD, Mylonakis E. Meta-analysis of methicillin-resistant Staphylococcus aureus colonization and risk of infection in dialysis patients. J Am Soc Nephrol. 2014;25:2131-41. doi: 10.1681/ ASN.2013091028.

4. Eells SJ, Kalantar-Zadeh K, Bolaris MA, May L, Miller LG. Body site Staphylococcus aureus colonization among maintenance hemodialysis patients. Nephron. 2015;129:7983. doi: $10.1159 / 000369348$.

5. Devraj A, Siva Tez Pinnamaneni V, Biswal M, Ramachandran R, Jha V. Extranasal Staphylococcus aureus colonization predisposes to bloodstream infections in patients on hemodialysis with noncuffed internal jugular vein catheters. Hemodialysis international International Symposium on Home Hemodialysis. 2017;21:35-40. doi: 10.1111/hdi.12450.

6. Zahed N-S, Aminzadeh Z, Akhavi Mirab AS, Gachkar L, Akhbari A. Nasal and extra-nasal methicillin resistant Staphylococcus aureus colonization among hemodialysis patients; is routine culturing of other body sites necessary? J Renal Inj Prev. 2018;7:280-5.

7. Sai N, Laurent C, Strale H, Denis O, Byl B. Efficacy of the decolonization of methicillin-resistant Staphylococcus aureus carriers in clinical practice. Antimicrobial resistance and infection control. 2015;4:56. doi: 10.1186/s13756-0150096-x.

\section{Financial Support}

This paper was not funded.

Copyright (c) 2019 The Author(s); Published by Published by Society of Diabetic Nephropathy Prevention. This is an open-access article distributed under the terms of the Creative Commons Attribution License (http://creativecommons.org/licenses/by/4.0), which permits unrestricted use, distribution, and reproduction in any medium, provided the original work is properly cited. 